\title{
A NOTE ON THE CONSTRUCTION OF QUASIFIELDS
}

\author{
N. L. JOHNSON
}

ABSTRaCt. Ostrom has extended certain finite nets to affine planes. In the present article, we extend Ostrom's results to the infinite case. We also develop a method of constructing quasifields and, in particular, a construction of semifields from given semifields. Some examples are also given.

1. Introduction. Ostrom [4] has extended certain finite nets to affine planes. We wish to first show that Ostrom's results do not require finiteness and then use the theory to construct (left) quasifields.

These quasifields $Q^{*}=(Q,+, *)$ are all of dimension 2 over a skewfield $F$ where $F$ is contained in the kernel of $Q^{*}$ and are constructed from ternary rings $Q=(Q,+, \cdot)$ where $Q$ is of dimension 2 over $F$. If $(Q,+, \cdot)$ is a (left) quasifield and $F$ is contained in the kernel of $Q$ then $Q^{*}$ is isomorphic to $Q$. If $(Q,+, \cdot)$ is a semifield then $(Q,+, *)$ is also a semifield.

In [3], Knuth has given a construction of four classes of finite semifields $S=(S,+, \cdot)$ where $S$ is of dimension 2 over a field $F$. Our construction method thus applies to these semifields. We will also show how to connect the four classes.

(1.1) Definition. Let $F$ be a skewfield and $V$ a 2-dimensional vector space over $F$.

Let $\Re$ : points $(x, y) \rightarrow x, y \in V$, lines $y=x \alpha+b, x=c, b, c \in V, \alpha \in F$. $\Re$ is said to be a derivable net.

(1.2) Definition. A transversal $T$ to a net $\mathscr{T}$ is a set of points of $\Re$ of the same cardinality as that of any parallel class $\rightarrow T$ intersects every line of $\Re$ exactly once.

(1.3) Definition. Let $x \rightarrow f(x)$ be a 1-1 correspondence $V \rightarrow V . f(x)$ is a transversal function iff

(i) $\forall c, d, c \neq d, f(c)-f(d), c-d$ are independent,

(ii) $\forall \alpha, b ; \alpha \in F, b \in V \exists c \in V \ni f(c)=c \alpha+b$.

(1.4) ThEOREM. If $T$ is a transversal of $\mathfrak{x}$ then there is a transversal function $f(x) \geqslant T=\{(x, y) \mid y=f(x)\}$. Conversely, every transversal function defines a transversal.

Proof. If $T$ is a transversal, (i) follows from Theorem 1 [4].

Received by the editors April 30, 1970.

AMS 1970 subject classifications. Primary 50D05; Secondary 05B25.

Key words and phrases. Quasifield, derivable nets, transversal, net extension, semifield. 
$\therefore\{(x, y) \mid y=f(x)\}=T$ satisfies (i). Moreover, $\{(x, y) \mid y=x \alpha+b\}$ $\cap T \neq \varnothing$. $\therefore(c, c \alpha+b)=(c, f(c))$ for some unique $c \in V$. $\therefore$ (ii) follows.

Conversely, if (i) and (ii) hold then (ii) shows that every line $l$ of $\Re$ intersects $\{(x, y) \mid y=f(x)\}$ in at least a point and (i) shows that $l \cap T$ is at most one point.

$\therefore\{(x, y) \mid y=f(x)\}$ intersects each line of $\mathscr{N}$ exactly once.

(1.5) Lemma. If $f(x)$ is a transversal function then $F(x)=f(x) \alpha+x \beta$ $+b$ is also a transversal function. $\forall \alpha \neq 0, \beta \in F, b \in V$.

Proof. Clearly by Lemma 1 [4], $F(x)$ satisfies (i).

Fix $\delta, d$. Choose any $\alpha \neq 0, \beta \in F$ and $b \in V$. Then consider $(\delta-\beta) \alpha^{-1}$, $(d-b) \alpha^{-1}$. By (ii), $\exists c \ni$

$$
\begin{aligned}
f(c) & =c\left((\delta-\beta) \alpha^{-1}\right)+(d-b) \alpha^{-1} \\
& \Rightarrow f(c) \alpha+c \beta+b=c \delta+d
\end{aligned}
$$

so $F(c)=c \delta+d$. Q.E.D.

(1.6) Theorem. If $T$ is a transversal to $\mathfrak{x}$ then there is an affine plane $\pi$ which contains $\Re$ and has $T$ as one of its lines.

Proof. The lines of $\pi=$ those of $\pi$ together with the transversals $f(x) \alpha+x \beta+b$.

By Theorem $2[4], \pi$ is at least a net. We must merely show that every pair of points of $\pi$ is joined.

Consider the points $(c, d)$ and $(r, m), c \neq r$. For each pair $\alpha, \beta \in F$ $\exists b, b$ (depending on $\alpha, \beta)$ э

$$
d=f(c) \alpha+c \beta+b, \quad m=f(r) \alpha+r \beta+b .
$$

(This is because every point is on exactly one line of each parallel class and each parallel-class slope is characterized by $(\alpha, \beta)$.)

We wish to show that $\exists$ a pair $\alpha, \beta$ э $b=b$.

Now

$$
d-m=(f(c)-f(r)) \alpha+(c-r) \beta+b-b .
$$

If $c=r$ or $d=m$ then the points $(c, d)$ and $(r, m)$ are joined. $\therefore$ Assume $c \neq r$. $\therefore f(c)-f(r)$ and $c-r$ are independent over $F$ so that $f(c)-f(r)$ and $c-r$ generate $V$ over $F$.

$\therefore \exists$ a unique pair $\alpha_{0}, \beta_{0} \in F_{7}(f(c)-f(r)) \alpha_{0}+(c-r) \beta_{0}=d-m$

$$
\Rightarrow b-b=0 \quad \text { for }\left(\alpha_{0}, \beta_{0}\right) \text {. }
$$

$\therefore b=b \quad$ for this pair $\left(\alpha_{0}, \beta_{0}\right)$. 
Thus, $\exists \alpha_{0}, \beta_{0}{ }^{\ni}$

$$
\begin{aligned}
d & =f(c) \alpha_{0}+c \beta_{0}+b \text { and } m=f(r) \alpha_{0}+r \beta_{0}+b \\
& \Rightarrow(c, d) \text { and }(r, m) \in\left\{(x, y) \mid y=f(x) \alpha_{0}+x \beta_{0}+b\right\} . \quad \text { Q.E.D. }
\end{aligned}
$$

(1.7) Theorem. The affine plane $\pi$ of (1.6) is a dual translation plane.

Proof. Ostrom's Theorems 3 through 7 [4] do not actually use finiteness so that we could appeal to this work.

However, it is also easy to see that (2.11) and (2.12) [5] are valid in the infinite case. Q.E.D.

\section{Construction of coordinate systems.}

(2.1) Theorem. Let $(Q,+, \cdot)$ be a ternary ring such that $(Q,+)$ is $a$ (right) 2-dimensional vector space over a skewfield $F$. Then, if $t \in Q-F$, we may define a new multiplication * as follows:

$$
a *(t \alpha+\beta)=(a \cdot t) \alpha+a \beta
$$

where the elements of $Q$ are represented in terms of the basis $\{1, t\}$ (where juxtaposition denotes skewfield multiplication).

Then

(i) $(Q,+, *)$ is a (left) quasifield whose kernel contains $F$;

(ii) if $\exists \bar{t} \in Q-F \ni(a+b) \cdot \bar{t}=a \cdot \bar{t}+b \cdot \bar{t} \forall a, b \in Q$ and if elements are represented in terms of the basis $\{1, \bar{t}\}$, then $(Q,+, *)$ is a semifield whose right nucleus contains $F$.

(See [3] for definitions.)

Proof. The function $f(x)=x \cdot t$ is a transversal function for the net $\mathscr{N}(Q)$ (see (1.1)). By (1.6) and (1.7) we thus have the proof of (i). Since the right distributive law follows directly from the assumptions of (ii), we also have the proof of (ii).

$Q=(Q,+, *)$ is a right vector space over its kernel $K$. Since $F \subseteq K$ either $Q$ is associative (1-dimensional over $K$ ) or the kernel of $Q$ is $F$.

(2.2) REMark. If $(Q,+, \cdot)$ is a ternary ring $\ni(Q,+)$ is a 2-dimensional vector space over a skewfield then if, for some $t \in Q-F$, $a \cdot(t+\alpha)=a \cdot t+a \alpha$ then $\left(Q,+, *_{t}\right)=\left(Q,+, *_{t+\alpha}\right)$. Thus, in particular if $(Q,+, \cdot)$ is a (left) quasifield and $K$ is the kernel of $Q$ and $K \subseteq F$ then $\left(Q,+, *_{t}\right)=\left(Q,+, *_{t \rho+\alpha}\right) \quad \forall \alpha \in F$ and $\forall \rho \in K$.

However, in general the systems $(Q,+, *)$ depend on the choice of $t$ and $\left(Q,+, *_{t}\right)$ is not in general isotopic to $\left(Q,+, *_{i}\right)$ (see (3.2)). Moreover, the corresponding planes may not be isomorphic. 


\section{Examples.}

(3.1) Example. Let $(s,+, \cdot)$ be a semifield such that $(s,+)$ is (right) 2-dimensional over a field $F$. Then $(\mathcal{S},+, *)(2.1)$ is a semifield whose right nucleus is contained in $F$.

(3.2) ExAmple. Let $(Q,+, \cdot)$ be a generalized André system (see [2]) $\rightarrow a \cdot b=a^{\sigma(b)} b$ where $\sigma(b)$ is a field automorphism of $\operatorname{GF}\left(q^{2}\right)$, $q=p^{r}, p$ a prime, depending on $b$ and such that $\sigma(\alpha)=\sigma(\beta)=1$ $\forall \alpha, \beta \in F=\mathrm{GF}(q)$ (see $[2,(2.2)]$ ). Then $(Q,+)$ is a right 2-dimensional vector space over $F$.

Let $t \in Q-F$. Then $a *(t \alpha+\beta)=\left(a^{\sigma(t)} t\right) \alpha+a \beta \forall a \in Q, \alpha, \beta \in F$ and $(Q,+, *)$ is a semifield whose right nucleus contains $F$.

Note that if $\sigma(t) \neq \sigma(\bar{t}),\left(Q,+, *_{t}\right)$ is not in general isomorphic or even isotopic to $\left(Q,+, *_{-}^{-}\right)$.

For example, if $\sigma(t)$ is the identity but $\sigma(\bar{t}) \neq \sigma(t)$ then $\left(Q,+, *_{t}\right)$ is a field and $\left(Q,+, *_{i}^{-}\right)$is in general a proper (nonassociative) semifield so that $\left(Q,+, *_{t}\right)$ and $\left(Q,+, *_{i}\right)$ are not isotopic and hence the corresponding affine planes coordinatized by these ternary rings cannot be isomorphic (see $[2,(4.2)]$ and $[3,(3.4 .3)]$ ).

(3.3) ExAmple. Let $(s,+, \cdot)$ be a semifield quadratic over a weak nucleus $\operatorname{GF}(q)$ (see $[3]$ ), then we can obtain a semifield $(s,+, *$ ). The system $D(S,+, *)$ dual to $(\delta,+, *)$ has the required properties of (2.1) so we may again construct a new *-system from $D(s,+, *)$. Therefore, we may construct a series of semifields by alternate applications of this extension process and dualization.

Knuth $[3, \S 7]$ has constructed the following classes of weak nucleus semifields.

Let $(Q,+)=\left(\mathrm{GF}\left(q^{2}\right),+\right), q=p^{r}, p$ a prime. In a weak nucleus semifield the multiplication $(t \alpha+\delta) \cdot(t \beta+\gamma), \alpha, \beta, \delta, \gamma \in \mathrm{GF}(q)$, $t \in Q-F=\mathrm{GF}(q)$, is determined whenever the multiplications $\alpha \cdot t$ and $(t \alpha) \cdot(t \beta)$ are known.

If $f \neq 0, g$ are constants in $\mathrm{GF}(q)=F$ and $\sigma \neq 1$ an automorphism of $F$ chosen so that $y^{\sigma+1}+f y-g=0$ has no solutions in $F$ then the following multiplications determine proper semifields $(Q,+, \cdot)$.

I. $\alpha t=t \alpha^{\sigma} ;(t \alpha)(t \beta)=t \alpha^{\sigma} \beta^{\sigma^{-1}} f+\alpha^{\sigma} \beta^{\sigma^{-2}} g$,

II. $\alpha t=t \alpha^{\sigma} ;(t \alpha)(t \beta)=t \alpha^{\sigma} \beta f+\alpha^{\sigma} \beta g$,

III. $\alpha t=t \alpha^{\sigma} ;(t \alpha)(t \beta)=t \alpha \beta^{\sigma^{-1}} f+\alpha^{\sigma^{-1}} \beta^{\sigma^{-2}} g$,

IV. $\alpha t=t \alpha^{\sigma} ;(t \alpha)(t \beta)=t \alpha \beta f+\alpha^{\sigma^{-1}} \beta g$.

(3.4) Theorem. Let $(Q,+, \cdot)$ be a Knuth semifield (K-semifield) of type I then $(Q,+, *)$ is a $K$-semifield of type II, the dual of $(Q,+, *)$, $(Q,+, \square)$ is a K-semifield of type III and the extension process on $(Q,+, \square)$ yields a $K$-semifield of type IV. 
Proof. If $(t \alpha) \cdot(t \beta)=t \alpha^{\sigma} \beta^{\sigma^{-1}} f+\alpha^{\sigma} \beta^{\sigma^{-2}} g$ then

$$
(t \alpha) *(t \beta)=((t \alpha) \cdot t) \beta=t \alpha^{\sigma} \beta f+\alpha^{\sigma} \beta g
$$

and

$$
\alpha * t=\alpha \cdot t
$$

so that $(Q,+, *)$ is of type II.

To dualize $(Q,+, *)$ merely interchange multiplications. That is, $a \square b=b * a$.

$$
\therefore t \square \alpha=\alpha * t=t \alpha^{\sigma}
$$

and

$(t \square \alpha) \square(t \square \beta)=\left(t \beta^{\sigma}\right) *\left(t \alpha^{\sigma}\right)=t \beta^{\sigma^{2}} \alpha^{\sigma} f+\beta^{\sigma^{2}} \alpha^{\sigma} g=t \square \beta^{\sigma} \alpha f+\beta^{\sigma^{2}} \alpha^{\sigma} g$

and

$$
\alpha \square t=t * \alpha=t \alpha=t\left(\alpha^{\sigma^{-1}}\right)^{\sigma}=t \square \alpha^{\sigma^{-1}} .
$$

Letting $\sigma=\sigma^{-1}$ we obtain a type III semifield.

Similarly the extension process on this type III yields a type IV.

Note that if the process were applied to II or IV no "new" semifield would be obtained since the right nuclei of the systems II and IV are initially both $\mathrm{GF}(q)$.

REMARKS. Bjork [1] has extended Ostrom's two-dimensional extension theory to the $n$-dimensional case. Apparently this work also does not require finiteness.

\section{REFERENCES}

1. G. Bjork, On finite nets associated with vector spaces, $\mathrm{Ph} . \mathrm{D}$. Thesis, Washington State University, Pullman, Wash., 1966.

2. D. A. Foulser, A generalization of Andre's systems, Math. Z. 100 (1967), 380395. MR 37 \#3436.

3. D. E. Knuth, Finite semifields and projective planes, J. Algebra 2 (1965), 182217. MR 31 \#218.

4. T. G. Ostrom, Derivable nets, Canad. Math. Bull. 8 (1965), 601-613. MR 33 $\$ 3185$.

5. - Vector spaces and construction of finite projective planes, Arch. Math. (Basel) 19 (1968), 1-25. MR 37 \#2081.

University of Iowa, Iowa City, Iowa 52240 